\title{
Percepción de un grupo de docentes de educación primaria acerca de la preparación recibida durante su formación universitaria en cuanto al tema de las Matemáticas
}

Perception of a group of elementary school teachers about their college math training

\section{Volumen 20, Número 1 \\ Enero - Abril \\ pp. 1-31}

Marianela Alpízar Vargas

Ana Lucía Alfaro Arce

\section{Citar este documento según modelo APA}

Alpízar Vargas, Marianela y Alfaro Arce, Ana Lucía. (2020). Percepción de un grupo de docentes de educación primaria acerca de la preparación recibida durante su formación universitaria en cuanto al tema de las Matemáticas. Revista Actualidades Investigativas en Educación, 20(1), 1-31. Doi. 10.15517/aie.v20i1.39978 


\title{
Percepción de un grupo de docentes de educación primaria acerca de la preparación recibida durante su formación universitaria en cuanto al tema de las Matemáticas
}

Perception of a group of elementary school teachers about their college math training

\author{
Marianela Alpízar Vargas ${ }^{1}$ \\ Ana Lucía Alfaro Arce ${ }^{2}$
}

\begin{abstract}
Resumen: Cada profesional confía en que lo aprendido en su proceso de formación inicial le será de utilidad en su vida laboral; sin embargo, la sociedad cambiante en la cual se vive hace que ese hecho sea volátil, en especial en carreras relacionadas con educación, ya que los cambios en la sociedad hacen que cambie el currículo educativo del país, por ello la formación inicial debe ser integral y dar las bases para autoformarse en caso necesario. Este artículo trata sobre la percepción que tiene un grupo de docentes, en ejercicio, de I y II ciclos de la Educación General Básica de Costa Rica acerca de su formación recibida en la universidad. La intención de reportar dicha percepción es concienciar a los entes que interfieren en la formación docente sobre los aspectos que se pueden mejorar. El estudio es cuantitativo de corte descriptivo, los datos se recolectaron por medio de un cuestionario aplicado a 121 docentes titulados en educación primaria de diversas regiones educativas del país en el año 2017. Sin importar el tipo de universidad donde se graduó, cada docente participante consideró que la formación inicial recibida no le ha sido suficiente para afrontar los retos del aula. El grupo de docentes participantes no se siente satisfecho con la formación recibida en sus casas de enseñanza porque consideran que algunas áreas del currículo actual, de Matemáticas, no fueron abordadas de la mejor manera, tanto en el conocimiento del contenido como en el didáctico. Por esta razón, indican que es urgente la necesidad de plantear procesos de formación continua para que puedan capacitarse en los temas que lo requieran. Los resultados obtenidos muestran que un grupo de docentes de Educación primaria en ejercicio no se encuentra satisfecho con la formación recibida en la universidad por lo que sería adecuado hacer una revisión de los planes de formación.
\end{abstract}

Palabras clave: Educación primaria, formación universitaria, matemáticas, procesos de capacitación.

\begin{abstract}
Professionals trust that what they have learned in their initial training will be useful in their working life. However, the changing society in which they live makes this a volatile fact, especially in careers related to education, since changes in society make the country's educational curriculum change. Consequently, college education must be comprehensive and provide a good foundation for self-formation, if necessary. This article is about the perception of a group of teachers of cycles I (1st to 3rd grade) and II (4th to 6th grade) of the General Basic Education of Costa Rica about their initial college education. Reporting about this perception has as aim to raise awareness, among the various actors that interfere in teacher training, about the aspects that can be improved in said training. The study was quantitative and descriptive. Data were collected from 121 elementary teachers from different educational regions in the country using a questionnaire in 2017. Regardless of the type of university from where they graduated, respondents felt that their initial college education had not been sufficient to meet classroom challenges. Teachers are not satisfied with their college education because they consider that some areas of the current Math curriculum were not addressed correctly, regarding content knowledge, as well as didactic content. The foregoing shows, on the one hand; the urgent need to establish professional development processes for these teachers to be trained in the required subjects; and on the other side, the urgent demand of making a Math curriculum upgrade of colleges.
\end{abstract}

Keywords: Elementary education, College education, Mathematics, profesional development courses.

${ }^{1}$ Académica de la Universidad Nacional en Escuela de Matemática, Costa Rica. Máster en Matemática Educativa del Centro de Investigaciones y de Estudios Avanzados del Instituto Politécnico Nacional de México. Dirección electrónica: marianela.alpizar.vargas@una.ac.cr ORCID http://orcid.org/0000-0002-3515-1971

${ }^{2}$ Académica de la Universidad Nacional (docencia, extensión, investigación) en Escuela de Matemática, Costa Rica. Magister en Estadística de la Universidad de Costa Rica. Dirección electrónica: ana.alfaro.arce@una.ac.cr ORCID https://orcid.org/0000-0002-9859-9474

Artículo recibido: 18 de junioi, 2019

Enviado a corrección: 10 de setiembre, 2019

Aprobado: 14 de octubre. 2019 


\section{Introducción}

La primaria es la base de la educación formal de las personas, en Costa Rica es parte de la Educación General Básica (EGB) comprende desde primero hasta sexto grado (I y II ciclos), su población cuenta con edades entre 6 y 12 años. En esta etapa se deben dar los cimientos para el aprendizaje que tendrán más adelante, en secundaria, y desarrollar competencias y habilidades que les permitan afrontar de manera adecuada las diversas situaciones de la vida cotidiana a las que se enfrentarán. Al ser esta etapa trascendental, es que se considera que el cuerpo docente, que tiene a cargo estos niveles educativos, debe poseer un perfil adecuado para encargarse de potenciar el desarrollo cognitivo de sus estudiantes (Programa Estado de la Nación, 2017). Tal como lo expresa Rebollero (2015), la calidad del sistema educativo en un país depende, en gran parte, de la buena formación que tenga su personal docente.

Chaverri y Sanabria (2010) señalan que el grupo de docentes de educación primaria influye directamente en la manera en que sus estudiantes ven el mundo, cómo lo interpretan y cómo se comportan. Por ende, es oportuno pensar que este grupo de profesionales debe tomar muy en serio su formación inicial en las aulas universitarias, así como su manera de enfrentarse a las situaciones que se les presenten al ejercer su profesión en los salones de clase, ya que su actuar influirá de manera directa sobre la vida de sus estudiantes.

Actualmente, en Costa Rica existen instituciones de educación primaria de índole públicas, privadas y subvencionadas, las públicas reciben la mayor cantidad de estudiantes. En el año 2016, 90,9\% del estudiantado de educación primaria asistió a centros educativos públicos (Programa Estado de la Nación, 2017).

El personal docente de las instituciones públicas es contratado directamente por el Ministerio de Educación Pública (MEP) a través del reclutamiento que realiza la Dirección General de Servicio Civil en conjunto con el Departamento del Personal del MEP. Los requisitos son de índole administrativo, tales como llenar formularios generales, presentar certificado de salud y de delincuencia, y el título universitario (Programa Estado de la Nación, 2008). A la universidad en donde obtuvo el título universitario no se le da importancia, sino solamente al grado académico que posea. Al mismo tiempo, no se solicita ningún perfil, ni se evalúan las competencias que tiene la persona oferente; es decir, se asume que la formación recibida en la universidad le será suficiente para impartir lecciones en la educación primaria, y esto es certificado por la misma universidad. 
En cuanto a las instituciones privadas, la manera en que contratan a su personal es variada, algunas seleccionan profesionales graduados en el área específica y otras escogen a estudiantes sin titulación, o profesionales en otras áreas, tales como preescolar o enseñanza de matemáticas, ciencias, estudios sociales o español, formados para educación secundaria, lo cual se evidencia en los resultados de este artículo.

La formación inicial del personal docente debe proporcionar las capacidades, habilidades y herramientas necesarias para que este enfrente de la mejor manera su papel en las aulas, considerando lo dinámica y cambiante que es la sociedad (Rebollero, 2015). Según Alpízar-Vargas y Alfaro-Arce (2019), en Costa Rica, no existen lineamientos generales para la formación de docentes de educación primaria, lo que hace que la variedad en los programas ofrecidos por las distintas universidades sea muy amplia. Esta situación no solo pasa en Costa Rica, en el estudio comparativo realizado por Rebollero (2015), se expresa que, en países europeos como Alemania, España, Francia, Reino Unido, Finlandia, entre otros, la diversidad en los planes de formación inicial de docentes de educación primaria es notoria, ya que, aunque se establecen indicadores comunes para el país, la decisión final de lo que se incluirá en su plan de formación lo decide cada universidad.

Los aspectos expuestos, anteriormente, dieron pie a realizar esta investigación, la cual trata de determinar la opinión que tiene el personal docente en ejercicio de educación primaria, en cuanto a la formación inicial que recibieron en la universidad, en el área de las matemáticas. Entre estos aspectos destaca: el papel trascendental de la educación en la niñez, la ausencia en el país de un perfil específico para la contratación del profesorado y el hecho de que los programas de formación no tengan un marco común.

De manera específica, el objetivo de este artículo es describir la percepción que tiene un grupo de docentes en ejercicio de I y I| ciclos de la EGB, en cuanto a su formación profesional, en el área de las matemáticas, en aspectos vinculados a los cursos universitarios recibidos, tales como: contenidos abarcados, metodología implementada, didáctica de las diversas áreas consideradas en los Programas de Estudio de matemáticas del MEP.

\section{Referente teórico}

Ruiz (2014) asegura que, a raíz de los cambios curriculares, en la disciplina de matemáticas en Costa Rica, el reto más importante que tiene el país es mejorar la preparación de su personal docente, ya que son la clave no solo de la implementación 
curricular, sino también de todo el progreso educativo. Por ende, se debe prestar atención tanto a la formación inicial recibida en las universidades como a la capacitación de docentes en servicio (citado en Alpízar-Vargas y Alfaro-Arce, 2019). Uno de los aspectos relevantes para prestar dicha atención es sobre el conocimiento (matemático y didáctico) que debe tener el personal docente que imparte las lecciones de matemáticas en educación primaria, por lo que en la siguiente sección se ahondará en este tema.

\subsection{Conocimiento de la persona docente de matemáticas en primaria}

Existen varios modelos que dan un acercamiento al tipo de conocimiento que debe poseer una persona para dar clases de matemática, sin importar el nivel de instrucción. A continuación, se citan algunos trabajos relacionados con esa temática. Shulman considera que el personal docente debe dominar distintas definiciones, conceptos y representaciones dentro de la disciplina que imparte, así como, estar en la capacidad de relacionar distintos conocimientos dentro de la disciplina y con otras áreas del saber; además, conocer la diversidad que presenta el estudiantado al cual le imparte lecciones (Shulman 1986, 1987). En 1986, hace su primera propuesta en la cual define tres categorías sobre el conocimiento profesional que debe tener quien imparte clases: conocimiento del contenido, conocimiento pedagógico del contenido y conocimiento curricular (Shulman, 1986). Para 1987, incluye otras categorías a su modelo: conocimiento pedagógico del contenido, conocimiento de estudiantes y sus características, conocimiento de los contextos educativos y conocimiento de los fines, propósitos y valores de la educación (Shulman, 1987).

A raíz del modelo establecido por Shulman, surge otro formulado por Ball, Thames y Phelps (2008) donde se caracteriza el conocimiento del profesorado de matemáticas en dos dominios: Conocimiento del Contenido y el Conocimiento Pedagógico del Contenido, cada dominio a su vez se subdivide en tres subdominios. Dicho modelo es conocido como Conocimiento Matemático para la Enseñanza (MKT por sus siglas en inglés).

El Conocimiento del Contenido enfoca lo referente a las matemáticas y se subdivide en el Conocimiento Común del Contenido (CCC, conocimiento de los contenidos que el profesorado tiene en común con otros profesionales que utilizan las matemáticas), Conocimiento en el Horizonte Matemático $(\mathrm{CH}$, conocimiento de la relación existente entre los conocimientos de matemáticas con otras áreas del currículo) y el Conocimiento Especializado del Contenido (CEC, conocimiento que se requiere para enseñar). Por su parte, el Conocimiento Pedagógico del Contenido, referido al proceso de enseñanza y 
aprendizaje de las matemáticas, se subdivide en Conocimiento del Contenido y los Estudiantes (CCEs, conocimiento que permite al personal docente interpretar el pensamiento de sus estudiantes respecto de las tareas y contenidos matemáticos), Conocimiento del Currículo (CC conocimiento acerca de la propuesta curricular) y Conocimiento del Contenido y la Enseñanza (CCEn, conocimiento que mezcla el saber sobre la enseñanza y sobre las matemáticas) (Ball et al., 2008).

De manera similar, Fennema y Franke (1992) consideran que la persona docente que imparte matemáticas no debe limitarse a conocer el contenido de la disciplina. Ellos propusieron un modelo donde establecen que, aparte del conocimiento disciplinar, deben poseer conocimiento pedagógico, de dicha disciplina, para poder utilizar diversas estrategias de enseñanza con una adecuada planificación y ejecución de la clase.

De lo anterior, se evidencia que múltiples autores coinciden en que una persona docente que imparte matemáticas, a cualquier nivel educativo, debe conocer acerca del contenido a enseñar, pero también debe saber acerca del conocimiento pedagógico o didáctico del mismo.

Por tanto, un programa de formación profesional en una carrera relacionada con la enseñanza debe considerar elementos tanto del Conocimiento del Contenido como del Conocimiento Pedagógico del Contenido, es decir, no quedarse solamente en los significados teóricos de los conocimientos, sino abarcar los elementos didácticos de estos. De manera similar, no es suficiente conocer diversas estrategias de enseñanza si no se tienen las bases teóricas del área a enseñar (Llinares, 2008).

En Costa Rica no existe ningún lineamiento relacionado con la formación de docentes que imparten las clases de matemática en educación primaria; esto indica que, no se tienen estándares definidos acerca del conocimiento que debe tener este tipo de profesional para integrarse al aula (Alpízar-Vargas y Alfaro-Arce, 2019). Lo anterior puede significar una limitante para el colectivo profesional en este campo, ya que depende de las decisiones que tome la universidad donde se inscriben.

Alfaro y Alpízar (2018) definen ciertas características que deben ser parte del perfil del personal docente de educación primaria, entre ellas: tener conocimiento especializado del contenido, así como conocimiento pedagógico de la disciplina, tener empatía con el estudiantado, entre otras; varias de estas características coinciden con lo expuesto por Ball et al. (2008). El y la docente, no solamente, deben conocer lo que van a desarrollar con el estudiantado, sino que deben contar con un conocimiento más especializado, saber muy 
bien cómo realizar la movilización de los conocimientos y en general sobre didáctica de las matemáticas. Además, debe ser un ente investigador de su propia práctica, estar anuente a la capacitación continua, conocer sobre herramientas tecnológicas que ayuden en el proceso de enseñanza y aprendizaje de las matemáticas y estar dispuesto a utilizarlas en las aulas, entre otras.

Con base en los elementos básicos que debe dominar una persona docente para impartir clases de matemáticas en primaria, es que se planteó esta investigación, con el fin de conocer la opinión de docentes en servicio, acerca de si estos elementos fueron abordados en las aulas universitarias mientras obtenían su título, en una carrera relacionada con educación básica.

\subsection{Formación inicial del personal docente}

La formación profesional que recibe el profesorado en las universidades debe centrarse en la necesidad de prepararlos para ser excelentes profesionales en su área; debe ser capaz de trasmitirles que el proceso de formación no se termina con la obtención del título universitario, sino que deben seguir actualizándose para ejercer de mejor manera la profesión docente (Llinares, 2008).

Chaverri y Sanabria (2010) consideran que no se le ha dado la importancia necesaria a los planes de estudio universitarios que forman docentes de I y II ciclos de la EGB. Acotan que el sistema de selección de personas candidatas es muy limitado, lo cual también se expresa en Alpízar-Vargas y Alfaro-Arce (2019), aunado al hecho de que no existe un perfil de ingreso y de egreso para las personas que estudian una carrera relacionada con educación primaria.

En el sexto Estado de la Educación se llama la atención sobre la urgencia de la delimitación de los perfiles de ingreso y salida de las personas vinculadas con el proceso de educación, y se sugiere que el Consejo Superior de Educación (ente encargado de aprobar los programas de estudio de la EGB) sea el que defina dichos perfiles (Programa Estado de la Nación, 2017). Asimismo, en la Política Educativa vigente en el país, se indica que se deben fortalecer los procesos de selección de las personas candidatas a ingresar a carreras relacionadas con educación, donde se contemplen aspectos como habilidades para comunicarse, aptitudes y actitudes acordes con la profesión docente (Consejo Superior de Educación, 2017). 
Según el sexto Estado de la Educación, el cuerpo de profesionales que están impartiendo clases en educación primaria, en el sector público, en general tiene altos grados académicos (licenciaturas y maestrías), trabajan tiempo completo en propiedad y se ubican en las categorías salariales más altas (alrededor de $70 \%$ de docentes de educación primaria son PT6) (Programa Estado de la Nación, 2017). Por lo anterior, se puede suponer que se cuenta con personal idóneo para cumplir con la labor docente; sin embargo, no se tienen controles sobre el trabajo que realizan en las aulas. Tal como lo citan Alpízar-Vargas y Alfaro-Arce (2019), en Costa Rica no existe una política educativa que establezca cuál es el perfil idóneo del docente de educación primaria para impartir matemáticas, así que tener títulos equivalentes no, necesariamente, significa contar con profesionales con niveles de conocimiento similares.

Chaverri y Sanabria (2010) consideran que la variabilidad existente entre la oferta de cada plan ofrecido por las universidades es muy amplia, en duración, cantidad de créditos y requisitos de graduación. Alpízar-Vargas y Alfaro-Arce (2019) agregan que hay dispersión en la cantidad de cursos por área de una universidad respecto a otra, y el perfil de docentes formadores es decidido por cada universidad y por ende variable.

Según el sexto Estado de la Educación, al analizar la trayectoria educativa de docentes en ejercicio, se evidencia que la mayoría de las licenciaturas y maestrías fueron obtenidas en universidades privadas $(58 \%$ de docentes de primaria en ejercicio se ha formado en instituciones privadas) (Programa Estado de la Nación, 2017). Estas universidades no tienen ninguna carrera acreditada por parte del Sistema Nacional de Acreditación de la Educación Superior (SINAES), relacionada con la formación general básica; es decir, docentes que impartirán las asignaturas: ciencias, estudios sociales, español y matemáticas.

En Costa Rica, el ente encargado de realizar evaluaciones de la calidad de las carreras universitarias es el SINAES, a través de la acreditación de estas. No obstante, no es obligación de las instituciones de educación superior someter sus carreras a esta evaluación, sino que es una decisión de cada institución. Por lo que un número importante de programas de formación no se someten a tal evaluación. Para el caso de educación básica en I y II ciclos, solamente las universidades estatales tienen sus carreras acreditadas (Alpízar-Vargas y Alfaro-Arce, 2019).

Según Alpízar-Vargas y Alfaro-Arce (2019) para el 2017, 27 instituciones de educación superior tenían aprobada al menos una carrera, relacionada con EGB; sin embargo, no todas 
ofrecieron ese año dichas carreras, solamente ocho instituciones privadas y tres públicas la ofertaron (información en la Tabla 1).

Tabla 1. Universidades estatales y privadas que ofrecieron carreras profesionales de Educación en I y II Ciclo de la EGB costarricense. Costa Rica, 2017

\begin{tabular}{|c|c|c|c|}
\hline & Universidad & Sigla & Carrera \\
\hline \multirow{3}{*}{ 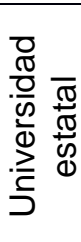 } & Universidad de Costa Rica & UCR & Bachillerato y licenciatura en Educación Primaria \\
\hline & Universidad Nacional & UNA & $\begin{array}{l}\text { Bachillerato y licenciatura en Pedagogía con énfasis en I y II Ciclo de } \\
\text { la EGB con salida lateral de diplomado }\end{array}$ \\
\hline & $\begin{array}{l}\text { Universidad Estatal a } \\
\text { Distancia }\end{array}$ & UNED & $\begin{array}{l}\text { Bachillerato y licenciatura en EGB I y II Ciclo con salida lateral de } \\
\text { diplomado }\end{array}$ \\
\hline \multirow{8}{*}{ 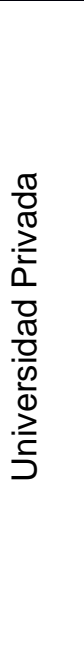 } & $\begin{array}{l}\text { Universidad de Cartago } \\
\text { Florencio del Castillo }\end{array}$ & UCA & $\begin{array}{l}\text { Bachillerato y licenciatura en Ciencias de la Educación con énfasis } \\
\text { en I y II Ciclo }\end{array}$ \\
\hline & $\begin{array}{c}\text { Universidad } \\
\text { Hispanoamericana }\end{array}$ & $\mathrm{UH}$ & $\begin{array}{l}\text { Bachillerato y licenciatura en Ciencias de la Educación con énfasis } \\
\text { En I Y II Ciclos de la EGB }\end{array}$ \\
\hline & $\begin{array}{l}\text { Universidad San Isidro } \\
\text { Labrador }\end{array}$ & UISIL & Bachillerato y licenciatura en Ciencias de la Educación I y II Ciclos \\
\hline & $\begin{array}{l}\text { Universidad Adventista de } \\
\text { Centro América }\end{array}$ & UNADECA & $\begin{array}{l}\text { Bachillerato y licenciatura en Ciencias de la Educación con énfasis } \\
\text { en I y II Ciclo de la EGB }\end{array}$ \\
\hline & $\begin{array}{l}\text { Universidad de las Ciencias } \\
\text { y el Arte }\end{array}$ & UNICA & Bachillerato y licenciatura en Educación con énfasis en I y II Ciclos \\
\hline & Universidad de San José & USJ & $\begin{array}{l}\text { Bachillerato en la Enseñanza Primaria con énfasis en Matemáticas } \\
\text { (Español, Inglés, Ciencias, Estudios Sociales) }\end{array}$ \\
\hline & Universidad Santa Lucía & USL & $\begin{array}{l}\text { Bachillerato y licenciatura en Ciencias de la Educación con énfasis } \\
\text { en I y II Ciclos }\end{array}$ \\
\hline & Universidad Federada & & $\begin{array}{l}\text { Bachillerato y licenciatura en Educación I y II Ciclo } \\
\text { Licenciatura en Educación I y II Ciclo }\end{array}$ \\
\hline
\end{tabular}

Fuente: Alpízar-Vargas y Alfaro-Arce (2019)

Sin hacer diferencias entre las universidades públicas o privadas, en los planes de formación de las carreras relacionadas con la EGB, se ofrecen cursos relacionados con filosofía, psicología, currículo, planeamiento, evaluación y didáctica general (Alfaro, Alpízar, Morales, Salas y Ramírez, 2013). La mayor parte de las mallas curriculares son destinadas a cursos relacionados con educación o pedagogía general. Todos los planes analizados cuentan con cursos relacionados con las asignaturas básicas: español, ciencias, estudios sociales y matemáticas, al menos uno relacionado con herramientas tecnológicas y, principalmente, a nivel de licenciatura, se ofrecen cursos dedicados a la investigación. Todas las universidades cuentan con práctica docente (Alpízar-Vargas y Alfaro-Arce, 2019).

En este estudio interesa la formación inicial universitaria que recibieron docentes de educación primaria en matemáticas, uno de los aspectos a considerar es la cantidad de cursos y creditaje, ya que algunos recibieron el doble de cursos y por ende la percepción hacia la disciplina podría ser distinta. 
Considerando las universidades que impartieron la carrera general (sin ningún énfasis) durante 2017, la de menor cantidad de créditos destinados para matemáticas es la UISIL, con dos cursos de tres créditos cada uno, seguido por la UNA con siete; mientras que los de mayor creditaje son UCR, UNADECA y UNICA, con 12 cada una. En cuanto a la cantidad de cursos, la UCR y UNADECA destinan la mayor cantidad con cuatro. Solamente, cinco de las universidades destinan dos cursos y en el caso de la UNA uno de los cursos no solo se destina a tópicos de matemáticas y educación matemática, sino que abarca temas de ciencias naturales (Alpízar-Vargas y Alfaro-Arce, 2019).

Algunas de las personas encargadas de la formación en el área de matemáticas en las universidades, tanto públicas como privadas, expresan que deben repasar muchos conceptos básicos en sus cursos, debido a que los y las estudiantes traen bases deficientes de secundaria (Alpízar-Vargas y Alfaro-Arce, 2019). Esto resulta preocupante porque, como se presentó anteriormente, es poco el tiempo que se dedica a la formación en matemáticas (según el total de cursos) y si se añade que se debe invertir tiempo en solventar esas debilidades, es evidente que no les alcanzará para ahondar en los significados y conceptos de los distintos contenidos, y en aspectos didácticos requeridos por cada área específica. Esto si se considera que el personal docente debe abarcar en su formación inicial, al menos las temáticas relacionadas con el currículo vigente, en el caso de Costa Rica: números, medidas, relaciones y álgebra, geometría y estadística, y probabilidad (MEP, 2012).

Tanto investigaciones nacionales como internacionales han externado que la formación inicial de docentes de primaria, en matemáticas, es un aspecto al que se le debe prestar especial atención debido a lo trascendental de las bases que se desarrollan en este nivel de enseñanza, entre ellos: Morín (2008), Jaimes - Duque, Rodríguez-Celis y Aguilar-Barreto (2016), Alfaro et al. (2013), Alpízar, Chavarría y Oviedo (2015), Chaves-Esquivel, AlpízarVargas y Alfaro-Arce (2016), Fernández (2018), Alpízar-Vargas y Alfaro-Arce (2019).

Según Morín (2008), el personal docente en formación tiene muchas deficiencias en didáctica de las matemáticas y en conceptos básicos de la disciplina que, a menudo, son acompañadas por una actitud negativa hacia dicha asignatura, lo cual puede perjudicar el proceso de enseñanza y aprendizaje del estudiantado que tendrán a cargo. Aunado a esto, Guzmán (2000, citado en Fernández, 2018) considera que la formación tanto en contenidos matemáticos como en aspectos didácticos, específicamente, de la asignatura de las matemáticas, que se recibe en la formación inicial del personal docente de primaria, es insuficiente para su labor de aula. 
En la investigación realizada por Morín, se pudo detectar que docentes que tenían un conocimiento adecuado del contenido matemático no tuvieron inconvenientes al momento de impartir la clase de matemáticas en cuanto a los conceptos desarrollados; no obstante, aquellos que reflejaron debilidades en dicho conocimiento tuvieron inconvenientes al impartirla. Aunado a ello, encontraron que sin importar el dominio del contenido disciplinar del personal docente participante del estudio, estos presentaron deficiencias en el conocimiento didáctico del contenido (Morín, 2008).

Las personas docentes de primaria no solo deben dominar aspectos teóricos de diversas asignaturas, sino también los aspectos didácticos y el desarrollo físico y emocional de sus estudiantes; por tanto, deben tener un vasto conocimiento acerca del contenido que van a enseñar y, a su vez, un conocimiento pedagógico o didáctico especializado. Según Alpízar-Vargas y Alfaro-Arce (2019), las universidades costarricenses que imparten carreras relacionadas con I y II ciclos están haciendo esfuerzos para desarrollar ambos conocimientos en futuros docentes, en algunas universidades es más evidente que se hacen esfuerzos compartidos, pero en otras existe un sesgo hacia la formación respecto al conocimiento pedagógico.

\subsection{Formación continua del personal docente}

La formación inicial del encargado de impartir las clases es fundamental para el éxito del proceso educativo. Sin embargo, es importante agregar que la formación continua de docentes en ejercicio es trascendental en este mismo proceso, ya sea para refrescar conocimientos disciplinares, actualizar estrategias metodológicas o para preparar al cuerpo docente para los cambios curriculares, más aún si la formación inicial no ha sido del todo satisfactoria. Cabe aclarar que los procesos de formación continua de docentes no sustituyen su formación inicial, solamente la complementan (Morales, 2017).

En los procesos de capacitación se identifican dos etapas en Costa Rica. La primera, lo ocurrido antes de la aprobación de los Programas de Estudio de matemáticas en 2012; la segunda, con la oferta de capacitaciones, que se ha dado a partir de ese momento. Morales (2017) indica que los planes de capacitación antes del 2012 no tenían mucha planificación, tenían poca duración y el seguimiento de estos no existía, lo cual concuerda con Venegas (2010, citado en el tercer Estado de la Educación) (Programa Estado de la Nación, 2011). A partir de 2012, el proyecto Reforma de la Educación Matemática en Costa Rica ha sido protagonista en los procesos de capacitación (Morales, 2017). 
Sumado a la capacitación docente existen eventos académicos en matemáticas que tienen participación de docentes de educación primaria, entre ellos: Festival Internacional de Matemática organizado, entre otras instituciones, por la Fundación para el Centro Nacional de la Ciencia y la Tecnología (CIENTEC) y por las universidades estatales, Congreso Internacional sobre la Enseñanza de la Matemática Asistida por Computadora (CIEMAC), Encuentro sobre Didáctica de la Estadística, la Probabilidad y el Análisis de Datos (EDEPA), organizados por el Instituto Tecnológico de Costa Rica (TEC) y Encuentro de Enseñanza de la Matemática por la UNED, el Congreso de Educación Primaria de la UCR y el Simposio de Educación Matemática en Primaria (SEMP) de la UNA.

De lo anterior, se evidencia un esfuerzo desde diversos enfoques y perspectivas que trata de contribuir en la formación continua de docentes que imparten matemáticas en la educación primaria, debido a que si no se tuvo la formación idónea en la universidad deben buscarse los medios para capacitarse y actualizarse. Además, tal como lo expresa Llinares (2008), la persona docente debe estar en formación constante.

\section{Metodología}

En este apartado se describe el enfoque del estudio realizado, así como la selección de participantes, la manera en que se recolectaron los datos, los instrumentos que se utilizaron en la recolección y, de manera general, el modo en que se analizaron los datos.

\subsection{Enfoque}

Esta investigación es de corte cuantitativo y se puede clasificar como no experimental descriptiva, ya que se describen las opiniones de un grupo de docentes tal cual fueron expresadas, sin ninguna intervención de las investigadoras, lo cual coincide con lo expuesto por Hernández, Fernández y Baptista (2006), quienes consideran que en este tipo de investigaciones se describen hechos, para los cuales se busca generar las interpretaciones correspondientes.

\subsection{Unidades de análisis}

Como población para este estudio se podría considerar a todo el personal docente en ejercicio de I y II ciclos de la EGB, de distintas zonas geográficas de Costa Rica, que tienen formación para impartir las asignaturas básicas en primaria (matemáticas, estudios sociales, español y ciencias) en educación pública, privada o subvencionada durante el año 2017. 
Debido a la cantidad de docentes que conforman la población, a la imposibilidad de las investigadoras de aplicar un cuestionario a dicha población y al objetivo general que tiene este estudio es que se trabajó con una muestra no probabilística, la cual se eligió a conveniencia. Dicha elección se dio debido a que el acceso a la población no es sencillo, y al propósito del estudio que es describir la percepción que tiene un grupo de docentes, en cuanto a su formación profesional, en el área de las Matemáticas, el cual no intenta dar la opinión general de la población docente del país ni tampoco generalizar resultados.

Para la aplicación del cuestionario se utilizaron diversas estrategias: aplicación en los centros educativos, privados y públicos de la Dirección Regional de Educación de Heredia. Se aplicó a los participantes del I SEMP, llevado a cabo por la Escuela de Matemática de la UNA en 2017. Por último, se construyó una versión digital del cuestionario y se envió por correo electrónico a algunos docentes de primaria de las diversas regiones educativas del país; para la obtención de las direcciones de correos electrónicos se recibió ayuda de colegas de la Escuela de Matemática de la UNA y de personas que fungen como asesores pedagógicos regionales de matemáticas del MEP, de manera aproximada se contactaron 300 personas y se logró tener respuesta de 132, de las cuales 114 fueron mujeres. En cada cuestionario se indicaba el fin de dicho instrumento; además, que se llenaba de manera voluntaria y que los resultados se usarían de maneja conjunta sin exponer casos particulares.

En 132 cuestionarios (92 en línea y 40 impresos), hubo representatividad de educación privada con 24 docentes, educación subvencionada con cuatro y educación pública con 104; asimismo, representación de 20 regiones educativas del país, de un total de 27 que existen y de todas las provincias de Costa Rica. Las investigadoras buscaron tener esa variabilidad en el tipo de institución, direcciones regionales y provincias del país, con el fin de obtener opinión de la mayor cantidad posible de docentes; sin embargo, esa representatividad no debe interpretarse, de ningún modo, como que esta investigación persiga generalizar los resultados a la población de interés.

Se excluyeron 11 cuestionarios porque las personas que lo completaron no contaban con un título universitario que las acreditara en carreras de I y II ciclos de la EGB. Por ende, y dado el interés de este estudio que es la percepción acerca de la formación universitaria en una carrera relacionada con educación básica, el análisis se efectuará con solamente 121 personas que cumplían con los requisitos establecidos, este grupo de 121 docentes se llamarán en adelante entrevistados o participantes del estudio. 


\subsection{Técnicas de recolección}

Los datos se recolectaron por medio de un cuestionario sobre percepción aplicado, durante el año 2017 a un grupo de docentes de primaria, el objetivo fue indagar acerca de los cursos que recibieron en el área de matemáticas y su didáctica durante los años de formación universitaria, en aspectos como: contenidos abarcados, metodología empleada y elementos relacionados con didáctica de las diversas áreas de los Programas de Estudio del MEP; además, de su percepción sobre la formación continua o capacitación recibida en los últimos años, este último tema no será considerado en este artículo.

Dicho cuestionario constaba de cinco partes. En la primera se indagaron aspectos generales en cuanto a la institución donde labora, título o títulos obtenido(s) y la universidad donde obtuvo el(los) título(s); la segunda parte incluyó contenidos que se abarcaron en los cursos que tuvieron que llevar durante su formación universitaria relacionados con el área de matemáticas; en la tercera parte se les consultó acerca de la metodología y de la evaluación llevada a cabo en los mismos cursos. En la cuarta parte se indagó acerca de la didáctica específica que abordaron y, por último, se preguntó acerca de los procesos de capacitación y actualización en los que han participado en los últimos cinco años, o en los que le gustaría participar. En total, el instrumento contenía 37 preguntas, en el cual se mezclaron cerradas y abiertas, prácticamente la totalidad de estas responden a variables con escala de medición nominal y ordinal.

En relación con la validez del instrumento, el cuestionario fue construido por las investigadoras, quienes se basaron, de manera específica, en la intención de reportar la opinión del grupo de docentes encuestado, referida a su formación inicial en la universidad, por lo que, a juicio de expertos, se incluyeron variables que permitieran medir de forma adecuada lo deseado.

Por otro lado, no se pretende categorizar al participante por el tipo de formación que recibió. Para determinar la confiabilidad del cuestionario, se calculó el alfa de Cronbach agrupando los ítems que se respondían con una misma escala y con el mismo sentido de respuesta. Se obtuvo en todos los casos la conclusión de que la consistencia interna de la escala utilizada es alta, puesto que los coeficientes del alfa resultaron superiores a 0,90. Como ejemplo, para los ítems con escala Siempre (5), Casi siempre (4), Algunas veces (3), Pocas veces (2) y Nunca (1), se obtuvo un alfa de Cronbach de aproximadamente 0,92. 


\subsection{Procesamiento de análisis}

Como el objetivo de este artículo es describir la percepción que tiene el grupo seleccionado de docentes en ejercicio en cuanto a su formación en las aulas universitarias, de manera específica en matemáticas, se realizó un análisis descriptivo con las opiniones que dieron por medio del cuestionario citado en el apartado anterior. Se utilizaron herramientas de estadística descriptiva para la presentación de los resultados, la información se despliega en tablas simples y de doble entrada. Asimismo, en gráficas que apoyan las afirmaciones que se hacen al respecto.

La presentación de los resultados se hizo por apartados: datos generales, cursos de matemáticas, metodología y evaluación de dichos cursos, y el papel de las didácticas específicas. Se agruparon y contabilizaron las respuestas dadas en cada pregunta del cuestionario. En algunos casos que compararon las respuestas, según el tipo de universidad de procedencia (pública o privada), esta comparación se hizo en términos relativos, no se realizaron pruebas de hipótesis, debido a que la muestra utilizada en el estudio no es aleatoria, y el objetivo de este no fue generalizar resultados a la población.

\section{Resultados}

En esta sección se describen las respuestas que dieron las personas encuestadas que cumplían con los requisitos del estudio, es decir los 121 participantes. Los resultados se van a exponer de acuerdo con los apartados que dividían el cuestionario, a saber: datos generales, cursos recibidos relacionados con matemáticas, metodología y evaluación de estos, y elementos relacionados con didáctica de las diversas áreas de matemática, presentes en los Programas de Estudio del MEP (números, geometría, álgebra y relaciones, medidas, probabilidad y estadística) recibidos durante su formación universitaria.

\subsection{Información general}

Casi $78 \%$ de las personas participantes cuentan con licenciatura o maestría en el área de interés, lo que da indicios de una preparación adecuada para impartir lecciones de las materias básicas en el nivel estudiado. Esta información coincide con lo expuesto en el sexto informe del Estado de la Educación, donde se expresa que la mayoría de docentes en servicio tienen grados académicos de licenciatura y maestría (Programa Estado de la Nación, 2017). Solamente cuatro docentes tienen como mayor grado un diplomado, lo cual representa una formación universitaria muy limitada, pues dicha titulación conlleva una 
duración de dos o tres años en su equivalente en cursos universitarios y solo fue o es ofrecido por las universidades estatales; por otra parte, 12 participantes no dijeron su mayor grado académico.

Para las investigadoras fue de interés conocer el tipo de universidad donde las personas participantes obtuvieron su mayor grado académico, para la presentación de estos datos se excluyen quienes cuentan solamente con un diplomado y aquellos que no dieron respuesta a la pregunta. En la Tabla 2 se puede observar la información donde se evidencia que la mayoría de participantes cuentan con licenciatura como mayor grado académico alcanzado, y que la mayor parte de quienes cuentan con una licenciatura como máximo la obtuvieron en una universidad privada.

Tabla 2. Costa Rica. Número de personas participantes por tipo de universidad según el mayor grado académico obtenido, 2017

\begin{tabular}{lccc}
\hline \multicolumn{1}{c}{ Universidad } & Bachillerato & Licenciatura & Maestría \\
\hline Universidades estatales & 7 & 19 & 2 \\
Universidades privadas & 4 & 46 & 17 \\
Universidades extranjeras & 0 & 1 & 0 \\
No contestó & 0 & 5 & 4 \\
\hline Total & $\mathbf{1 1}$ & $\mathbf{7 1}$ & $\mathbf{2 3}$ \\
\hline
\end{tabular}

Fuente: Elaboración propia, 2017

Por otra parte, una misma persona puede tener título universitario de bachillerato, licenciatura y maestría, en una carrera relacionada con educación en I y II ciclos. De las personas participantes de este estudio, 105 cuentan con bachillerato universitario y 94 con licenciatura. A nivel de bachillerato, la UNED y la UNA tienen la mayor cantidad de personas graduadas, con alrededor de 48\% (acumulado entre las dos); mientras que, a nivel de licenciatura, la UISIL tiene el mayor porcentaje con aproximadamente $21 \%$, seguida por la UCA y la UNA. La variabilidad en la oferta de la universidad donde obtuvieron los distintos grados académicos es muy alta, en el caso de bachillerato citaron tres universidades estatales y 14 privadas; mientras que para licenciatura citaron las mismas estatales y 13 privadas. Cabe aclarar que para 2017, de las universidades nacionales citadas, siete no estaban ofertando carreras relacionadas con educación básica.

En cuanto a la obtención del título de maestría, afín a la carrera en estudio, 23 docentes afirmaron contar con este, solamente dos lo obtuvieron en una universidad estatal (UNA) y la mayor cantidad de graduados la tiene la UISIL (5 personas). 
Se evidencia en este estudio lo estipulado por el sexto Informe del Estado de la Educación, donde se establece que una parte importante del personal docente obtiene su bachillerato en una universidad estatal y luego obtiene su licenciatura o maestría en una universidad privada (Estado de la Nación, 2017), esto también coincide con lo expuesto por Alpízar-Vargas y Alfaro-Arce (2019) donde comunican que algunas universidades privadas tienen un incremento en la matrícula de estudiantes de licenciatura que provienen de realizar el bachillerato en alguna de las estatales. A nivel de bachillerato 54\% lo obtuvo en una universidad estatal; mientras que la licenciatura (en este tipo de universidad) solo $29 \%$ y la maestría, 9\%.

Como se evidenció en el marco conceptual de este estudio, sin importar el tipo de universidad, se ofrecen cursos de matemáticas en los planes de estudio. En algunos casos, solamente se da uno, y en las que mayor cantidad ofertan, se imparten cuatro; esto ha ido cambiando debido a las actualizaciones que realizan las universidades con miras a mejorar. En el caso de las estatales, la acreditación y la actualización las obliga a estar en constantes revisiones y cambios, mientras que en las universidades privadas no hay ninguna regulación que las obligue a actualizar sus planes, pero la competencia y los cambios que se han dado en los Programas de Estudio del MEP hace que muchas de ellas se actualicen. A continuación, se presentan las respuestas dadas por las personas participantes de este estudio en preguntas relacionadas con la cantidad de cursos, en el área de matemáticas, que recibieron y algunas particularidades de estos.

\subsection{Cursos de matemáticas}

Alrededor de $81 \%$ de las personas entrevistadas afirmó haber recibido durante su formación universitaria cursos donde se incluían contenidos de matemáticas o de su didáctica. No obstante, preocupa que 10 personas afirmen no haber tomado cursos relacionados con esta disciplina, de ellas, cuatro se encontraban en el 2017 impartiendo clases de matemáticas (los seis restantes no contestaron la pregunta).

Las investigadoras consideraron que podría ser de interés separar a los y las participantes por tipo de universidad y comparar las opiniones, sin embargo; al indagar sobre la procedencia de la universidad donde obtuvieron cada título académico se evidenció una variabilidad muy amplia debido, como se citó anteriormente, a que una cantidad considerable de docentes obtienen su bachillerato en una modalidad y la licenciatura en otra. Por tanto, al hacer esa división solamente 64 docentes, del total de los y las participantes, se pudieron 
clasificar como graduados o graduadas de una modalidad específica (es decir hicieron su bachillerato y licenciatura en el mismo tipo de universidad), de estos, 37 son graduados de educación privada.

Al hacer la clasificación por tipo de institución no hay mucha diferencia con la muestra completa, ya que $89 \%$ afirmó haber recibido cursos de Matemática o Matemática Educativa y si se clasifican por universidad, $86 \%$ de los graduados de universidades privadas apuntan que recibieron cursos de matemáticas, mientras que en las públicas es de $89 \%$. Es importante aclarar que la muestra de docentes que completó el cuestionario no se eligió de manera aleatoria, por lo que no se pueden realizar pruebas estadísticas para determinar diferencias significativas, este estudio lo único que hace es comparar porcentualmente los datos. Además, se destaca que en las universidades consideradas en las investigaciones de Alfaro et al. (2013) y Alpízar-Vargas y Alfaro-Arce (2019) se impartía al menos un curso relacionado con matemáticas, por tanto, las personas que expresaron lo contrario en esta encuesta, pueden haber obtenido su titulación en universidades distintas a las incluidas en los estudios citados.

Alrededor de $47 \%$ de las personas participantes recibió, durante su formación universitaria, solamente uno o dos cursos de matemática o matemática educativa. Se asume que en estos se contemplaron tanto los conocimientos teóricos de la disciplina como sus fundamentos didácticos; no obstante, deja en duda si uno o dos cursos son suficiente para la formación del personal docente que desarrolla el currículo de matemáticas en educación primaria. Además, tal como lo expresan Alpízar-Vargas y Alfaro-Arce (2019), algunas de las personas encargadas de impartir los cursos de matemáticas de las carreras de educación básica consideran que dos cursos son insuficientes para la formación que deben tener sus graduados y graduadas para afrontar con éxito su labor de aula.

El 66\%, de 98 participantes que expresaron haber llevado al menos un curso de matemáticas en su formación universitaria, afirmó que el enfoque de los cursos incluyó aspectos teóricos y didácticos de esta disciplina. Este es un aspecto positivo, ya que indica que no solamente se está prestando atención al conocimiento del contenido, sino también al conocimiento pedagógico del contenido, lo cual coincide con Ball et al. (2008) y su modelo MKT. Lo expresado por el grupo encuestado también coincide con Alpízar-Vargas y AlfaroArce (2019) cuando mencionan que en las instituciones de educación superior se están llevando a cabo esfuerzos para desarrollar ambos dominios en los docentes en formación. 
De las personas que afirmaron que los cursos tuvieron solamente un componente teórico (12 en total), seis obtuvieron su bachillerato en una universidad estatal, mientras que $21,4 \%$, de 98 participantes, afirmó que su formación fue orientada solamente en el enfoque didáctico.

De los y las participantes que aseguraron haber recibido al menos un curso de matemáticas, la mayoría afirmó que estos fueron recibidos a nivel de bachillerato o entre el diplomado y el bachillerato, lo cual coincide con el estudio realizado por Alpízar-Vargas y Alfaro-Arce (2019) donde se evidencia que la mayoría de centros de enseñanza universitaria tiene en sus mallas curriculares los cursos relacionados con matemáticas en bachillerato y en cuanto a las estatales, UNA y UNED los tienen también a nivel del diplomado.

\subsection{Contenidos teóricos elementales de matemáticas}

Luego de determinar que 98 participantes si habían recibido cursos de matemáticas o matemática educativa, se les consultó si consideraban que los contenidos abarcados fueron suficientes para su accionar docente, más de $61 \%$ consideró que no (60 participantes). Esto concuerda con lo indicado por Alpízar-Vargas y Alfaro-Arce (2019), ellas expresan que personas encargadas en carreras de formación docente en primaria y algunos docentes de esas carreras, consideran que la formación en esta área ha quedado corta de acuerdo con las demandas actuales. El sentimiento del personal docente participante en cuanto al hecho de que los contenidos abarcados no fueron suficiente se comparó, según la universidad de procedencia.

De los 98 que recibieron al menos un curso, solamente 56 docentes se pudieron clasificar por el tipo de universidad donde se formaron (si sacaron el bachillerato y la licenciatura en el mismo tipo de universidad), los resultados se muestran en la Tabla 3.

Tabla 3. Número y porcentaje de personas participantes por tipo de universidad según si los contenidos matemáticos fueron suficientes para impartir clases de matemática en primaria, Costa

Rica, 2017

\begin{tabular}{|c|c|c|c|c|c|}
\hline \multirow[t]{3}{*}{ Tipo de universidad } & \multicolumn{5}{|c|}{$\begin{array}{l}\text { Los contenidos matemáticos abarcados en los cursos } \\
\text { universitarios fueron suficientes para impartir clases de } \\
\text { matemática en primaria }\end{array}$} \\
\hline & \multicolumn{2}{|c|}{ Sí } & \multicolumn{2}{|c|}{ No } & \multirow{2}{*}{ Total } \\
\hline & Absoluto & $\%$ & Absoluto & $\%$ & \\
\hline Privada & 11 & 34 & 21 & 66 & 32 \\
\hline Pública & 8 & 33 & 16 & 67 & 24 \\
\hline Total & 19 & 34 & 37 & 66 & 56 \\
\hline
\end{tabular}

Fuente: Elaboración propia, 2017 
No se detectan diferencias, en puntos porcentuales, de la opinión entre participantes provenientes de universidades privadas o públicas en cuanto a que la formación recibida en matemáticas es insuficiente para afrontar su labor profesional. Lo que sí es evidente es el descontento que muestran docentes en ejercicio en cuanto a lo abarcado en las aulas universitarias, esto debe llamar la atención a los entes formadores y al MEP. No solo es necesario cambiar los planes de estudio de las carreras en la actualidad, sino que se deben establecer planes de formación continua que permitan llenar los vacíos que percibe el personal docente en servicio. Lo anterior concuerda con la Política Educativa vigente en Costa Rica, la cual indica que se deben fortalecer las relaciones existentes entre las diversas entidades que participan en el proceso de educación como entes formadores, MEP, entre otros, con miras a robustecer el sistema educativo (Consejo Superior de Educación, 2017).

Respecto a los planes de formación continua, existen diversos intentos por parte de las universidades y el MEP de ofrecer espacios de capacitación, actualización y discusión, tales como los citados en el marco conceptual de este artículo; sin embargo, hace falta una mayor oferta. Además, es necesario que se detecten las necesidades específicas para atacarlas con mayor ahínco, esto para que los procesos sean valorados por los y las docentes. Adicionalmente, es importante que se realicen seguimientos a quienes participan de estos procesos.

En la Tabla 4 se enlistan algunas de las razones que mencionaron las personas participantes, disconformes con su formación, para justificar que no tienen una preparación adecuada. Cabe recordar que la cantidad de docentes disconformes con su formación en esta área es 60 , ellos podían expresar diversos motivos, por lo que en la tabla siguiente no aparece el total de docentes. 
Tabla 4. Número de docentes participantes que consideran que la formación recibida durante la universidad en Matemáticas no es la idónea, según motivo mencionado. Costa Rica, 2017

\begin{tabular}{lc}
\hline \multicolumn{1}{c}{ Motivos } & $\begin{array}{c}\text { Cantidad de } \\
\text { docentes }\end{array}$ \\
\hline $\begin{array}{l}\text { Los temas que se tratan en las aulas universitarias son muy simples o se abarcan de } \\
\text { manera superficial }\end{array}$ & 23 \\
\hline $\begin{array}{l}\text { El énfasis dado al curso no estuvo en las estrategias metodológicas ni didácticas } \\
\text { específicas de las matemáticas para usar en el aula }\end{array}$ & 15 \\
\hline $\begin{array}{l}\text { Los temas abarcados no corresponden a los presentes en los Programas de Estudio del } \\
\text { MEP vigentes }\end{array}$ & 14 \\
\hline La realidad del aula es distinta, cantidad de estudiantes, adecuaciones curriculares, etc. & 8 \\
\hline $\begin{array}{l}\text { La falta de preparación del docente que imparte el curso tanto a nivel de temáticas de } \\
\text { matemáticas como metodologías de enseñanza y uso de herramientas tecnológicas }\end{array}$ & 5 \\
\hline El poco tiempo para la cantidad de contenidos & 4 \\
\hline La escases de práctica en de aula & 4 \\
\hline El curso estuvo orientado a didáctica general y no se abarcaron contenidos propios del & 4 \\
\hline área de matemáticas & 4 \\
\hline
\end{tabular}

Fuente: Elaboración propia, 2017

Los principales motivos para considerar que su formación en matemáticas no fue la ideal fueron: que los temas abarcados en los cursos universitarios fueron simples o se abarcaron de manera superficial, no se trabajaron diversas estrategias metodológicas y no se abordó la didáctica específica de la mejor manera. Esto puede deberse al poco tiempo que dedica la mayor parte de los planes de formación para matemáticas. Además, casi una cuarta parte consideró que en sus cursos de formación no se abarcaron todos los temas que en la actualidad se encuentran presentes en los Programas de Estudio del MEP. Esto puede responder a que los programas en vigor fueron aprobados en 2012 y varios encuestados se graduaron antes de esa fecha. Sin embargo; actualmente algunos programas de formación cuentan con cursos de matemática que, por lo menos en el planteamiento de su descriptor, no cubren las cinco áreas presentes en dichos programas (Alpízar-Vargas y Alfaro-Arce, 2019).

De los motivos mencionados por las personas participantes se encuentran tanto los relacionados con el conocimiento del contenido, así como los concernientes con el conocimiento pedagógico del contenido. Entre ellos, se pueden citar: los contenidos son abarcados de manera superficial, o no se cubren ni siquiera los definidos en los Programas de Estudio del MEP; no se abordaron de manera correcta los elementos de didáctica de las matemáticas que son indispensables para la formación docente. Esto último, coincide con lo reportado por Alfaro y Alpízar (2018) donde asesores y asesoras pedagógicas y personas 
encargadas de la dirección de instituciones de educación primaria expresan que la formación ofrecida por las universidades en matemáticas ha sido escasa.

Cinco participantes expresaron que la persona docente formadora que tuvieron a cargo no tenía la preparación necesaria para impartir el curso respectivo, esto resalta porque, como lo expresan Alpízar-Vargas y Alfaro-Arce (2019), no hay ningún lineamiento establecido acerca de la persona responsable de formar, en matemáticas, a futuros docentes de educación primaria. En algunas universidades lo hace alguien graduado de enseñanza de las matemáticas con formación o sensibilización para educación primaria, mientras que, en otras, los cursos son impartidos por personas con titulación en educación primaria, sin formación en matemáticas ni en su didáctica.

En el currículo se establecen cinco áreas matemáticas que deben desarrollarse a lo largo de la EGB, a saber: números, geometría, medidas, relaciones y álgebra y, estadística y probabilidad. Por lo anterior, en el cuestionario se realizó un desglose por área para priorizar aquellas donde la formación estuvo escasa o que se deba prestar mayor atención para la oferta de capacitación. Se dividió en conocimiento del contenido y conocimiento pedagógico o didáctico de cada área, los resultados se expresan a continuación.

\subsection{Conocimiento del contenido}

Se realizó una división de las áreas específicas a abordar para conocer qué tan acertada fue la formación de las personas participantes en dichas áreas. Para cada una de ellas debían indicar el nivel de profundidad con el que recibieron los contenidos, solamente fueron considerados los 98 participantes que aseguraron haber recibido al menos un curso de matemáticas durante su formación.

Tabla 5. Número y porcentaje de personas participantes según nivel de profundización con el que abordaron las áreas temáticas presentes en los Programas de Estudio del MEP en la formación universitaria, Costa Rica, 2017

\begin{tabular}{|c|c|c|c|c|c|c|c|c|c|c|c|c|c|c|}
\hline \multirow{3}{*}{$\begin{array}{c}\text { Nivel de } \\
\text { profundización }\end{array}$} & \multicolumn{14}{|c|}{ Área de las matemáticas presentes en los Programas de Estudio del MEP } \\
\hline & \multicolumn{2}{|c|}{ Números } & \multicolumn{2}{|c|}{ Medidas } & \multicolumn{2}{|c|}{ Álgebra } & \multicolumn{2}{|c|}{ Relaciones } & \multicolumn{2}{|c|}{ Geometría } & \multicolumn{2}{|c|}{ Estadística } & \multicolumn{2}{|c|}{ Probabilidad } \\
\hline & Abs & $\%$ & Abs & $\%$ & Abs & $\%$ & Abs & $\%$ & Abs & $\%$ & Abs & $\%$ & Abs & $\%$ \\
\hline Mucho & 53 & 54 & 36 & 37 & 13 & 13 & 13 & 13 & 41 & 42 & 25 & 26 & 24 & 24 \\
\hline Poco & 42 & 43 & 49 & 50 & 54 & 55 & 53 & 54 & 47 & 48 & 49 & 50 & 46 & 47 \\
\hline Nada & 2 & 2 & 12 & 12 & 29 & 30 & 30 & 31 & 9 & 9 & 23 & 23 & 27 & 28 \\
\hline No contestó & 1 & 1 & 1 & 1 & 2 & 2 & 2 & 2 & 1 & 1 & 1 & 1 & 1 & 1 \\
\hline Total & 98 & 100 & 98 & 100 & 98 & 100 & 98 & 100 & 98 & 100 & 98 & 100 & 98 & 100 \\
\hline
\end{tabular}

Fuente: Elaboración propia, 2017 
La única área en la que más de $50 \%$ de docentes consideró que fue abarcada con un nivel de profundización alto fue números, este hecho es preocupante, ya que, para las otras, un alto porcentaje de los y las participantes afirmó que se abordó con poco o nada de profundidad. Álgebra y relaciones, se encuentran en el peor panorama, alrededor de 30\% considera que no se dio ningún grado de profundización. Esto puede deberse a que la inclusión del álgebra y las relaciones es nueva en el currículo de primaria del sistema educativo costarricense. Sin embargo; medidas y geometría, son temas que tienen longevidad en el currículo, y alrededor de 50\% aseguró que se profundizó poco en el estudio de estas.

En cuanto a estadística y probabilidad, más de $70 \%$ de los entrevistados considera que se le dio muy poca o nada de profundidad, estas temáticas se incluyeron en el currículo costarricense de primaria desde 1995, por ende, no hay motivo para que después de 20 años se encuentre un porcentaje tan alto de docentes que exprese que no se le dio una profundidad adecuada al tratamiento de estas temáticas durante su formación universitaria.

Cabe rescatar que la pregunta fue dirigida de manera general, no se establece aquí el tipo de profundización; por ejemplo, si ahondaron en el significado del concepto del objeto matemático o si solo realizaron diversas operaciones básicas con números reales 0 ejercicios rutinarios relacionados con los conceptos básicos, esto debido a que la profundización que recibieron en el área números pudo haber sido procedimental y no conceptual.

Por otra parte, se consultó sobre el área que consideraban que debería tener más énfasis en la formación del personal docente, es importante aclarar que la pregunta limitaba las respuestas, ya que debían asignar distintas prioridades a las áreas, por ello se obtuvo mucha diversidad en las respuestas, sin embargo; se puede destacar que un porcentaje alto de docentes (44\%) considera como prioritaria el área de números, lo cual coincide con el énfasis que ponen los Programas de Estudio al señalar que esta ocupa una posición predominante en los dos primeros ciclos (MEP, 2012). No obstante, debe prestarse atención al hecho de que no se trata de realizar operaciones básicas con números racionales de manera mecánica, sino de enfatizar en un sentido práctico de los números y sus propiedades, se debe buscar que estos sean significativos para la vida cotidiana y que tengan sentido de utilidad para el estudiantado (Alfaro y Alpízar, 2018).

Por su parte, llama la atención probabilidad, ya que $40 \%$ considera que tiene que recibir el menor énfasis en la formación, lo cual parece contradictorio con resultados 
obtenidos por Chavarría y Oviedo (2013), ellas trabajaron con una muestra de docentes de educación primaria, y como parte de las conclusiones expresan que la mayor parte de estos mostraron poco dominio de conceptos claves de probabilidad, agregan que esto puede deberse a la ausencia de conocimientos en esa área, pues la formación recibida en la universidad no fue suficiente.

Otro de los elementos considerados fue el grado de satisfacción que tenían respecto al desarrollo teórico elemental de los contenidos matemáticos, por cada área, en su formación universitaria. En la Tabla 6 se presentan los resultados.

Tabla 6. Número y porcentaje de docentes participantes según el grado de satisfacción de la formación recibida en la universidad sobre el contenido matemático. Costa Rica, 2017

\begin{tabular}{|c|c|c|c|c|c|c|c|c|c|c|c|c|c|c|}
\hline \multirow{3}{*}{$\begin{array}{l}\text { Grado de } \\
\text { satisfacción }\end{array}$} & \multicolumn{14}{|c|}{ Área de las matemáticas presentes en los Programas de Estudio del MEP } \\
\hline & \multicolumn{2}{|c|}{ Probabilidad } & \multicolumn{2}{|c|}{ Estadística } & \multicolumn{2}{|c|}{ Medidas } & \multicolumn{2}{|c|}{ Algebra } & \multicolumn{2}{|c|}{ Relaciones } & \multicolumn{2}{|c|}{ Geometría } & \multicolumn{2}{|c|}{ Números } \\
\hline & Abs & $\%$ & Abs & $\%$ & Abs & $\%$ & Abs & $\%$ & Abs & $\%$ & Abs & $\%$ & Abs & $\%$ \\
\hline Muy satisfecho & 10 & 10,2 & 13 & 13,3 & 12 & 12,2 & 7 & 7,2 & 9 & 9,1 & 16 & 16,3 & 27 & 27,6 \\
\hline Satisfecho & 26 & 26,5 & 24 & 24,5 & 26 & 26,5 & 19 & 19,4 & 20 & 20,4 & 31 & 31,6 & 29 & 29,6 \\
\hline satisfecho & 26 & 26,5 & 30 & 30,6 & 31 & 31,6 & 24 & 24,5 & 24 & 24,5 & 26 & 26,5 & 27 & 27,6 \\
\hline Poco satisfecho & 21 & 21,4 & 17 & 17,3 & 22 & 22,5 & 31 & 31,6 & 30 & 30,6 & 18 & 18,4 & 13 & 13,2 \\
\hline Nada satisfecho & 15 & 15,4 & 13 & 13,3 & 6 & 6,2 & 17 & 17,3 & 15 & 15,4 & 7 & 7,2 & 2 & 2,0 \\
\hline No contestó & 0 & 0 & 1 & 1,0 & 1 & 1,0 & 0 & 0 & 0 & 0 & 0 & 0 & 0 & 0 \\
\hline TOTAL & 98 & 100 & 98 & 100 & 98 & 100 & 98 & 100 & 98 & 100 & 98 & 100 & 98 & 100 \\
\hline
\end{tabular}

Fuente: Elaboración propia, 2017

Es un motivo de preocupación que solamente en números, la mayoría (57\%) se encuentra satisfecha con la formación recibida, seguido por geometría, mientras que las áreas con mayor insatisfacción son álgebra con, aproximadamente, 49\% y relaciones con $46 \%$ de participantes con poco o con nada de satisfacción. Lo anterior hace un llamado vehemente a las casas de enseñanza superior para revisar sus planes de formación en matemáticas, ya que al menos el grupo de personas que participaron en este estudio no se encuentra satisfecho con lo que recibieron.

Resulta desconcertante que desde hace varios años se presenten investigaciones donde se hace este llamado de atención; por ejemplo, en Alpízar, Chavarría y Oviedo (2015) se advierte que es necesaria una revisión de los cursos relacionados con matemáticas, que se encuentran en los planes de estudio de las distintas universidades que forman docentes de I y II ciclos, debido a que lo desarrollado en los diversos cursos no es suficiente para que 
el personal docente se sienta preparado para desarrollar sus clases. Pero; el presente estudio, denota un grupo de docentes que no ha visto mucha diferencia.

Alpízar-Vargas y Alfaro-Arce (2019) realizan un estudio sobre los planes de formación de las universidades que en 2017 impartieron carreras relacionadas con docentes de EGB y determinaron que algunos planes tienen más de 20 años de antigüedad, lo cual indica que esas universidades no han considerado los cambios del currículo costarricense de primaria para revisar sus mallas curriculares; aunque hay que hacer la salvedad de que, sí existen algunas que se han sometido a procesos de evaluación y actualización.

\subsection{Conocimiento pedagógico o didáctico del contenido}

Al igual que con los contenidos matemáticos, se les consultó acerca del grado de importancia que había tenido la didáctica específica, por área, para la enseñanza de las matemáticas en su formación. Para efectos de esta investigación el grado de importancia se considerará como si se cubrieron elementos de didáctica específica para cada área estipulada en los Programas de Estudios del MEP; por ejemplo, dificultades y errores de los estudiantes por cada temática, evaluación, métodos y estrategias de enseñanza, etc. Nuevamente los y las participantes considerados para el análisis de esta variable son los 98 que contestaron de manera afirmativa haber recibido cursos relacionados con matemáticas y su enseñanza. En la Tabla 7 se pueden observar los datos respectivos.

Tabla 7. Número y porcentaje de docentes participantes según el grado de importancia que se le dio a la didáctica específica en los cursos universitarios. Costa Rica, 2017

Área de las matemáticas presentes en los Programas de Estudio del MEP

\begin{tabular}{lcccccccccccccc}
\hline & \multicolumn{8}{c}{ Área de las matemáticas presentes en los Programas de Estudio del MEP } \\
\cline { 2 - 17 } $\begin{array}{l}\text { Grado de } \\
\text { importancia }\end{array}$ & Números & \multicolumn{3}{c}{ Medidas } & Álgebra & \multicolumn{2}{c}{ Relaciones } & Geometría & Estadística & \multicolumn{3}{c}{ Probabilidad } \\
\hline Mucho & 62 & 63 & 12 & 12 & 17 & 17 & 12 & 12 & 51 & 52 & 25 & 26 & 20 & 21 \\
Poco & 30 & 31 & 16 & 16 & 57 & 58 & 62 & 63 & 40 & 41 & 55 & 56 & 57 & 58 \\
Nada & 3 & 3 & 1 & 1 & 22 & 23 & 22 & 23 & 5 & 5 & 16 & 16 & 19 & 19 \\
No contestó & 3 & 3 & 69 & 71 & 2 & 2 & 2 & 2 & 2 & 2 & 2 & 2 & 2 & 2 \\
Total & 98 & 100 & 98 & 100 & 98 & 100 & 98 & 100 & 98 & 100 & 98 & 100 & 98 & 100 \\
\hline
\end{tabular}

Fuente: Elaboración propia, 2017

Para números, $63 \%$ consideró que se dieron suficientes elementos didácticos para la enseñanza de esta temática. En geometría, más de 50\% afirmó lo mismo, sin embargo; para álgebra, relaciones, estadística y probabilidad, la mayor parte consideró que fue poco el énfasis y poca la importancia al conocimiento didáctico del contenido o a la didáctica 
específica de la temática. Este aspecto vuelve a llamar la atención, ya que según el modelo MKT es importante que el cuerpo docente tenga una buena formación en aspectos didácticos vinculados de manera directa con el área a impartir. En las carreras relacionadas con educación básica, se imparten muchos cursos de índole general sobre pedagogía, didáctica y educación para infantes (Chaverri y Sanabria, 2010), pero es indispensable y fundamental que se retomen aspectos propios de las matemáticas y su didáctica. El área de medidas no se tomó en cuenta para el análisis, ya que un porcentaje muy alto de participantes (71\%) no contestó la pregunta.

Se les consultó acerca de los tópicos que se incluyeron en los diferentes cursos relacionados con aspectos pedagógicos o didácticos de las matemáticas. En la Figura 1 se presenta el detalle, debe considerarse que en un mismo curso se pudieron abordar diversos aspectos pedagógicos, por ende una persona docente podía citar varios de estos.

Figura 1. Número de participantes según los tópicos relacionados con Didáctica de las Matemáticas que se abordaron durante la formación universitaria. Costa Rica, 2017

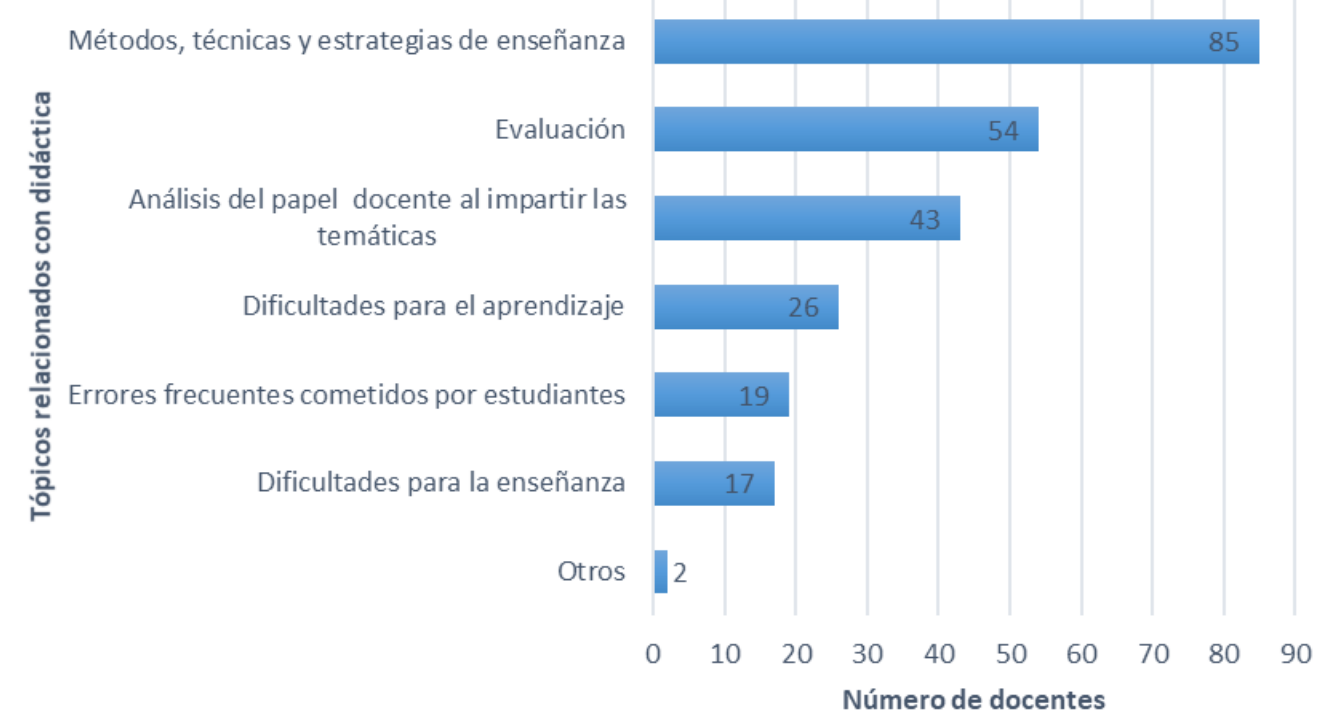

Fuente: Elaboración propia, 2017

El tópico que la mayoría afirmó haber recibido fue Métodos, técnicas y estrategias de enseñanza, seguido por Evaluación, estos son los tradicionales en un proceso de formación, además de indispensables; sin embargo, en los últimos años se ha venido concienciando sobre la importancia de conocer acerca de las dificultades y los errores del estudiantado para poder planificar de mejor manera el proceso de enseñanza y aprendizaje. Tal como lo 
expresa Lupiáñez (2013), las limitaciones para el aprendizaje pueden hacer más lento o hasta frenar por completo este proceso en el estudiantado. Cabe aclarar que esta pregunta es general, es decir, puede que los métodos, técnicas y estrategias de enseñanza, se enfocaran solamente en una de las áreas; por ejemplo, en números y no se abarcara nada en relación con las otras.

Al consultarles acerca del grado de satisfacción que tenían respecto a la formación recibida en cuanto al contenido didáctico, la mayoría de las personas se encuentra satisfecha con lo recibido en números (55\%). Para todas las demás áreas, los resultados mostraron que la mayoría de personas docentes se concentran en las categorías de mediana satisfacción y ausencia de esta. $50 \%$ no se encuentra satisfecho con la formación en álgebra, $48 \%$ en relaciones, $43 \%$ en estadística y $44 \%$ en probabilidad. En la Tabla 8 se presenta el desglose de los datos.

Tabla 8. Número y porcentaje de docentes participantes según el grado de satisfacción con la formación universitaria recibida en didáctica de cada una de las áreas de las Matemáticas presentes en el currículo oficial. Costa Rica, 2017

\begin{tabular}{lccccccccccccccc}
\hline & \multicolumn{8}{c}{ Área de las matemáticas presentes en los Programas de Estudio del MEP } \\
\cline { 2 - 16 } \multicolumn{1}{c}{$\begin{array}{c}\text { Grado de } \\
\text { satisfacción }\end{array}$} & \multicolumn{1}{c}{ Números } & \multicolumn{1}{c}{ Medidas } & $\%$ & Abs & $\%$ & Abs & $\%$ & Abs & $\%$ & Abs & $\%$ & Abs & $\%$ & Abs & $\%$ \\
\hline Muy satisfecho & 28 & 28,6 & 6 & 19,4 & 11 & 11,2 & 10 & 10,2 & 22 & 22,4 & 17 & 17,4 & 15 & 15,3 \\
$\begin{array}{l}\text { Satisfecho } \\
\text { Medianamente }\end{array}$ & 26 & 26,5 & 7 & 22,6 & 11 & 11,2 & 12 & 12,2 & 23 & 23,5 & 17 & 17,4 & 18 & 18,4 \\
satisfecho & 26 & 26,5 & 10 & 32,2 & 27 & 27,6 & 29 & 29,6 & 27 & 27,6 & 22 & 22,4 & 22 & 22,4 \\
Poco satisfecho & 15 & 15,3 & 8 & 25,8 & 35 & 35,7 & 31 & 31,6 & 21 & 21,4 & 26 & 26,5 & 26 & 26,5 \\
Nada satisfecho & 3 & 3,1 & 0 & 0 & 14 & 14,3 & 16 & 16,4 & 5 & 5,1 & 16 & 16,3 & 17 & 17,4 \\
TOTAL & 98 & 100 & 31 & 100 & 98 & 100 & 98 & 100 & 98 & 100 & 98 & 100 & 98 & 100 \\
\hline
\end{tabular}

Fuente: Elaboración propia, 2017

Las personas docentes no solo deben manejar conceptos elementales del área a enseñar, sino que es importante que conozcan acerca de la didáctica específica de cada una de las temáticas que deben desarrollar en sus aulas, ya que no se enseñan de la misma manera.

Por ejemplo, en geometría se requiere un incentivo en la ubicación espacial y en la visualización, mientras que en números son necesarios: la agilidad y el cálculo mental, así como el dominio de algoritmos, etc. Según este estudio existe un grupo de personas docentes que considera que el desarrollo que se le dio en la universidad a estas temáticas fue insuficiente, esto conlleva a dos panoramas: el primero, donde el personal docente es 
autodidacta y trata de autoformarse, al leer o buscar capacitación externa; y el segundo donde no lo considera importante y su accionar con el estudiantado se limita a la exposición, memorización y uso de libros de texto, sin ningún tipo de reflexión ni análisis.

Solamente $31 \%$ asegura estar satisfecho con la formación general recibida en la universidad, pues considera que esta le permitió desarrollarse de manera adecuada en su campo laboral. Este dato es muy desalentador, ya que la formación universitaria debe dar las bases y fundamentos sólidos, no solamente en el conocimiento del contenido a enseñar, sino también en el conocimiento pedagógico o didáctico. Por su parte, 69\% de las personas encuestadas consideraron que su formación inicial no ha sido suficiente para afrontar su labor en las aulas, esto alarma la situación, ya que si la persona docente no se encuentra preparada para impartir las lecciones en un área podría ser que les refleje a sus estudiantes una actitud negativa hacia dicha área, o que la enseñanza de los conocimientos específicos, no la realice de la mejor manera al no sentirse con la preparación para hacerlo.

\section{Conclusiones}

Según el objetivo general de esta investigación, el cual enfatizaba en describir la percepción de un grupo de docentes en ejercicio de I y II ciclos de la EGB en cuanto a la preparación recibida, en su formación inicial en la universidad podemos rescatar que, sin importar la procedencia de la universidad (privada o pública), las personas encuestadas señalan que la formación inicial que recibieron no ha sido suficiente para afrontar los retos educativos actuales. Entre las razones que citan están: estuvieron hace muchos años en la universidad y el currículo ha cambiado desde entonces, no se le dio importancia al desarrollo de los distintos conocimientos que requiere el personal docente para impartir clases en educación primaria.

Este último aspecto mencionado por las personas participantes en el estudio es de gran relevancia, puesto que hace alusión a los diversos tipos de conocimientos que debe dominar el personal docente, según Shulman y otros investigadores, señalados en el referente teórico.

Lo anterior hace concluir que es necesario que las universidades encargadas de la formación realicen un análisis de su oferta académica y actualicen sus programas. Según Alpízar-Vargas y Alfaro-Arce (2019), solamente algunas de las universidades que imparten carreras relacionadas con I y II ciclos han hecho algún análisis de sus planes y los han cambiado después de la aprobación de los Programas de Estudio de matemáticas del MEP 
en 2012, algunas universidades tienen planes de formación con más de 20 años de antigüedad.

Por lo general, la cantidad de cursos dedicados a las matemáticas y a su didáctica es a lo sumo de dos, ya sea en universidad pública o privada. La cantidad de conocimientos que debe tener un docente, que imparte matemáticas, en educación primaria, es amplia por lo que es necesario que la oferta curricular contemple mayor cantidad de tiempo dedicado a esta asignatura y no, solamente, hacia la cobertura de contenidos básicos y operatorios, sino hacia el abordaje conceptual y didáctico de los mismos. Con este estudio se pudo constatar que al menos el grupo de docentes en servicio participante, considera que la formación inicial que tuvo fue insuficiente; hallazgo concordante con las conclusiones de Fernández (2018) y, Alpízar-Vargas y Alfaro-Arce (2019) relacionadas con que la formación, tanto en contenidos matemáticos como en aspectos didácticos, específicamente de la asignatura de las matemáticas, que se recibe en la formación inicial del personal docente de primaria, es insuficiente para su accionar en las aulas.

Es necesario crear un perfil de ingreso de estudiantes para carreras relacionadas con educación en I y II ciclos de EGB, el cual debe ser planteado y revisado por las universidades, que ofrecen estos planes de formación. Así como, un perfil del egresado de dichas carreras, ya que la variabilidad con la que se egresan es muy amplia, y el principal empleador (el MEP) pone muy pocos controles sobre la formación recibida. Dicho perfil debe estar vinculado con el currículo costarricense y debe contener aspectos relacionados con el conocimiento del contenido matemático y con el conocimiento pedagógico o didáctico, tal como lo establecen Ball et al. (2008).

Como fue expresado en el referente teórico de este artículo el profesorado no solo debe dominar contenidos disciplinares del área de las matemáticas, sino que debe saber y aplicar diversas estrategias didácticas para lograr que sus estudiantes comprendan los tópicos. Algunas universidades han hecho esfuerzos importantes por integrar estos dos componentes en sus planes de formación (Alpízar-Vargas y Alfaro-Arce, 2019), pero según la opinión de las personas participantes de este estudio es necesario que las casas de formación presten mayor atención a la integración de la disciplina con los aspectos didácticos que tratan la enseñanza de la misma. Por ejemplo, se debe trabajar en aspectos como: conocimiento del contenido a enseñar de manera conceptual y operacional; tipos de aprendizaje, dificultades y errores que tienen los estudiantes; así como maneras de gestionar una clase para lograr aprendizaje en sus estudiantes. 
La mayor parte de las personas encuestadas afirman no estar satisfechas con la formación académica que recibieron en la universidad. El área de números es la que califican con mejor puntuación, pero, según los y las participantes del estudio, otras áreas como relaciones, álgebra, probabilidad y estadística, fueron dejadas de lado en los planes de formación que completaron.

Las casas de enseñanza superior deben asumir su responsabilidad con sus egresados y egresadas ofreciendo procesos de capacitación y actualización porque, al menos los encuestados aseguran estar insatisfechos con la formación recibida en el área de las matemáticas y en aspectos didácticos de ella. Dichos procesos deberían estar articulados y tratar de manera eficiente los temas donde el cuerpo docente tiene más necesidad e interés. Por ende, es importante seguir con investigaciones relacionadas con el conocimiento que tienen las personas docentes en ejercicio en ciertas áreas de las matemáticas, y así poder ofertar los cursos idóneos.

Esta investigación da a conocer que un grupo de docentes no está satisfecho con la formación recibida en su universidad; sin embargo, no se consideraron otras variables como la cantidad de años que han transcurrido desde que se graduó para determinar si este factor condicionaría la opinión, y si los esfuerzos hechos por algunas universidades en los últimos años han fructificado. Por tanto, esta línea de investigación debe seguirse para conocer el estado actual de la formación en matemáticas desde la perspectiva de la persona docente y plantear posibles soluciones.

\section{Agradecimientos o reconocimientos}

Trabajo elaborado en el marco del proyecto de investigación Formación docente en II Ciclo de la Educación General Básica en cuanto al tema de las Matemáticas de la Escuela de Matemática de la Universidad Nacional, bajo el código SIA 0082-16.

\section{Referencias}

Alfaro, Ana Lucía y Alpízar, Marianela. (2018). Perfil del docente que imparte matemáticas en la educación primaria: visión de diversos actores (Informe 2, Proyecto Formación docente en II Ciclo de la Educación General Básica en cuanto al tema de las Matemáticas). Heredia, Costa Rica: UNA.

Alfaro, Ana Lucía; Alpízar, Marianela; Morales, Yuri; Salas, Oscar, y Ramírez, Melvin. (2013). La formación inicial y continua de docentes de matemáticas en Costa Rica. Cuadernos de Investigación y Formación en Educación Matemática, 8 (número especial), 131179. Recuperado de http://revistas.ucr.ac.cr/index.php/cifem/issue/view/1281 
Alpízar-Vargas, Marianela y Alfaro-Arce, Ana Lucía. (2019). La formación universitaria de docentes de educación primaria: el caso de matemáticas. Uniciencia, 33(2), 110-154. doi: doi.org/10.15359/ru.33-2.8

Alpízar, Marianela; Chavarría, Laura y Oviedo, Katalina. (2015). Percepción de un grupo de docentes de I y II ciclo de educación general básica de escuelas públicas de Heredia sobre los temas de estadística y probabilidad. Revista Actualidades Investigativas en Educación, 15(1), 1-23. doi: DOI 10.15517/AIE.V15I1.17728

Ball, Deborah; Thames, Mark y Phelps, Geoffrey. (2008). Content Knowledge for Teaching: What Makes It Special? Journal of Teacher Education, 59(5), 389-407. doi: doi.org/10.1177/0022487108324554

Chavarría, Laura y Oviedo, Katalina. (2013). Actitudes y conocimientos que tienen docentes de la Educación General Básica costarricense de I y II Ciclo sobre Estadística y Probabilidad, en la Dirección Regional Educativa de Heredia (tesis de licenciatura inédita). Universidad Nacional, Heredia, Costa Rica. Recuperado de http://www.matematica.una.ac.cr/index.php/documentacion-digital/category/12-tesis

Chaverri, Diego y Sanabria, Marcela. (2010). Estudio comparativo entre los programas que ofrecen tres universidades públicas y siete universidades privadas en la carrera de I y II ciclo de la educación general básica en Costa Rica. Revista Actualidades Investigativas en Educación, 10(3). 1-33. doi: DOI 10.15517/AlE.V10I3.10145

Chaves-Esquivel, Edwin; Alpízar-Vargas, Marianela y Alfaro-Arce, Ana Lucía. (2016). Percepción de los docentes de primaria en ejercicio, acerca de las matemáticas y su enseñanza en relación con los programas oficiales del MEP. Uniciencia, 30(1), 31-55. doi: doi.org/10.15359/ru.30-1.3

Consejo Superior de Educación. (2017). Política Educativa: La persona: centro del proceso educativo y sujeto transformador de la sociedad. San José, Costa Rica: Ministerio de Educación Pública.

Fennema, Elizabeth y Franke, Megan. (1992). Teachers' knowledge and its impact. In D.A. Grouws (Eds), Handbook of Research on Mathematics Teaching and Learning ( $\mathrm{p}$. 147-164). New York: The National Council of Teachers of Mathematics.

Fernández, Ceneida (2018). Proyecto docente e investigador. Informe de investigación. Universidad de Alicante.

Hernández, Roberto, Fernández, Carlos y Baptista, Pilar. (2006). Metodología de la Investigación (4a.ed.). México: McGraw-Hill Interamericana.

Jaimes-Duque, Dany, Rodríguez-Celis, Teófilo y Aguilar-Barreto, Andrea. (2016). Lineamientos de la enseñanza de la matemática y la formación docente. Revista Eco.Mat., 7(1), 61-70. doi: doi.org/10.22463/17948231.1104 
Llinares, Salvador. (2008). Construir el conocimiento necesario para enseñar matemática. Prácticas Sociales y Tecnología Evaluación e Investigación, 1(3), 7-30. Recuperado de http://www.saber.ula.ve/handle/123456789/27786

Llinares, Salvador. (2012). Formación de profesores de matemáticas. Caracterización y desarrollo de competencias docentes. Cuadernos de Investigación y Formación en Educación Matemática, 7(10), 53-62. Recuperado de https://revistas.ucr.ac.cr/index.php/cifem/article/view/10559

Lupiáñez, Jose Luis. (2013). Análisis Didáctico: La planificación del aprendizaje desde una perspectiva curricular. En Luis Rico, Jose Luis Lupiáñez, y Marta Molina (Eds.), Análisis Didáctico en Educación Matemática: Metodología en investigación, formación de profesores e invención curricular (pp. 81-101). Granada, España: Editorial Comares, S.L.

Ministerio de Educación Pública (MEP). (2012). Programas de Estudio en Matemáticas para la Educación General Básica y el Ciclo Diversificado. San José, Costa Rica: autor.

Morales, Yuri. (2017). La formación continua de docentes de matemáticas para un cambio del currículo a nivel nacional: el caso de Costa Rica (2012 - 2015). Perspectivas da Educação Matemática - INMA/UFMS, 10(23), 453-473. Recuperado de http://seer.ufms.br/index.php/pedmat/article/view/4480

Morín, Marie-Pier. (2008). Les connaissances mathématiques et Didactiques chez les futurs maîtres du primaire: quatre cas à l'étude. Canadien Journal of Education 31(3), 537566. doi: $10.2307 / 20466715$

Programa Estado de la Nación en Desarrollo Humano Sostenible. (2008). Estado de la Educación 2. San José, Costa Rica: Consejo Nacional de Rectores (CONARE).

Programa Estado de la Nación en Desarrollo Humano Sostenible. (2011). Estado de la Educación 3. San José, Costa Rica: Consejo Nacional de Rectores (CONARE).

Programa Estado de la Nación en Desarrollo Humano Sostenible. (2017). Estado de la Educación 6. San José, Costa Rica: Consejo Nacional de Rectores (CONARE).

Rebollero, Teresa. (2015). La formación inicial del profesorado de educación primaria y secundaria en Alemania, España, Finlandia, Francia y Reino Unido. Estudio Comparado. Revista Española de Educación Comparada, 25, 129-148. doi: $\underline{10.5944 / \text { reec.25.2015.14787 }}$

Shulman, Lee. (1986). Those Who Understand: Knowledge growth in Teaching. Educational Researcher, 15(2), 4-14. doi: http://dx.doi.org/10.3102/0013189X015002004

Shulman, Lee. (1987). Knowledge and Teaching: foundations of the New Reform. Harvard Educational Review, 57(1), 1-22. doi: http://dx.doi.org/10.17763/haer.57.1.j463w79r56455411 
Revista indizada en

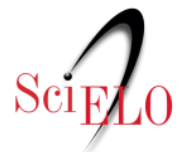

redalyc satindex

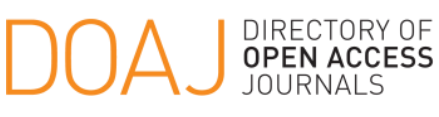

Distribuida en las bases de datos:

- Dialnet

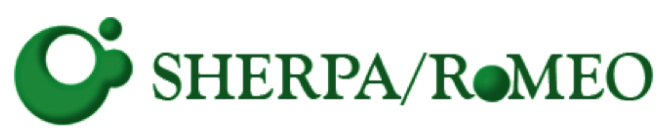

REDIB

Red Iberoamericana

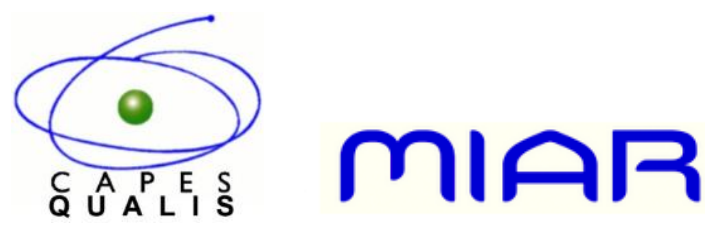

